\title{
Ansteckungen, Spaltungen, Variationen \\ zum Schreiben oder Töten - Erzählte Suizide seit Werther
}

Contagion, Partition, Variation on Writing and Killing: Suicide Narrations since Werther

Stephan Kurz

\begin{abstract}
Suicide narrations have been viewed through the lens of Goethe's landmark novel. This paper, grounded in studying the epistolary novel genre, aims at enhancing knowledge about this text's impact on both the generic and the motif successions in (German language) literature by looking into the narrative modes employed in depictions of writing and killing, both of which are singular moments of subjectivity. Examples for later variations on the suicide motif are mainly taken from Fin-de-Siècle literature which in particular expands the means of narration, while the broad concept of textual interrelations remain unquestioned.
\end{abstract}

\section{Keywords}

suicide, genres and motifs, epistolary novel, intertextuality 


\section{Einleitung}

Die Leiden des jungen Werthers ist der kanonische Ausgangstext für eine Auseinandersetzung mit dem Suizid in der deutschsprachigen Erzählliteratur. Die folgenden Miszellen beziehen sich zunächst auf das 18. Jahrhundert, mit gattungsspezifischen und thematischen Weiterungen zum Werther. Die folgenden Überlegungen gehen teilweise von den Ergebnissen der Dissertationsschrift des Verf. aus, ${ }^{1}$ sind aber einerseits erweitert um neue Erkenntnisse hinsichtlich des Briefromans von Herbert Croft, und andererseits um die Vergleichspunkte zu »Fin-de-Siècle-Suiziden« (Pippo Spano und Lieutnant Gustl).

An Goethes Roman messen sich einerseits der Briefroman als Gattung, andererseits aber auch alle literarischen Texte mit Suizid-Thematik nach 1774. Beiden Bereichen soll im Folgenden Rechnung getragen werden. Vorweg auszuschließen gilt es den »Werther-Effekt « ${ }^{2}$ und eine moralische Bewertung des Suizids. Demgegenüber hält sich das Vorliegende an die Frage nach der Semantisierung von Suizid sowie nach seiner kulturhistorischen Bedeutung. Thomas Macho spricht von einer »Kulturtechnik des Suizids «, ${ }^{3}$ die abhängig ist von den Medien ihrer Vermittlung, und mit Foucault und Hadot auch von einer »Selbsttechnik «. ${ }^{4}$ Das Folgende bleibt innerhalb der Grenzen der Fiktion - berücksichtigt sind Geschichten der Intertextualität und nicht der Nachahmung literarischer Suizide in der außerliterarischen Realität.

Vorauszusetzen ist an der Grenze zum echten Leben dann doch auch der therapeutische Effekt von niedergeschriebenen Suizidphantasien, den Goethe in Dichtung und Wahrheit andeutet, ${ }^{5}$ also das Rettende am Schreiben, die andere Seite von »ums Leben schreiben«: Schreiben über eine Selbsttechnik zur Verhinderung einer Selbsttechnik als Selbsttechnik. Weiters ist vorauszusetzen, dass der Werther nur einer von hunderten Romanen ist, die die Gattung Briefroman hervorgebracht hat. ${ }^{6}$

In einem ersten Schritt soll eine mögliche Engführung der Subjektspaltung in Narration und Suizid thematisiert werden, um zweitens über das Scharnier des Gattungsbegriffs zu dem prototypischen Suizid-Briefroman Werther zu kommen, der dann drittens

1 Verf., Gebundene Korrespondenzen. Gattungs- und Mediengeschichte von Briefromanen des 18. Jahrhunderts, Wien, dazu zuletzt ders., Der Briefroman des 18. Jahrhunderts als Gattungsproblem, in: Jahrbuch: Das Achtzehnte Jahrhundert und Österreich, 2018.

2 Für die historische Lektürenkonstellation von 1774 bis etwa 1800 hat Martin Andree, Wenn Texte töten: Über Werther, Medienwirkung und Mediengewalt, München 2006 gerade 12 direkte Werther-Suizide belegt; in der Suizidologie ist der Werther-Effekt dennoch eine Größe.

3 MACHO, Thomas: Das Leben nehmen: Suizid in der Moderne, Erste Auflage, Berlin 2017, S. 17. Machos Buch spannt einen großen Bogen vom Suizid in der Antike bis zu rezenten Sterbehilfedebatten.

4 ebd, 92.

5 Vgl. Johann GOETHE, Wolfgang: Goethes Werke [Sophienausgabe], hg. v. im Auftrag der Großherzogin Sophie von Sachsen, unveränd. Nachdr. d. Ausg. Weimar, Böhlau, 1890, Weimar 1999, Bd. 28, Dreizehntes Buch, S. 220: Goethe »beschloß, zu leben. Um dieß aber mit Heiterkeit thun zu können, mußte ich eine dichterische Aufgabe zur Ausführung bringen, wo alles, was ich über diesen wichtigen Punct empfunden, gedacht und gewähnt, zur Sprache kommen sollte«.

6 Die Sekundärliteratur allein zu Werther ist schwer zu überblicken, Vollständigkeit ist hier daher nicht zu behaupten. 
selbst als Ausgangstext für in der einen (formalen) oder der anderen (thematischen) Hinsicht kommensurable literarische Texte erscheint.

\section{Ums Leben schreiben: Subjektspaltung in Narration und Suizid}

Der Roman Die Leiden des jungen Werthers offeriert eine Passionsgeschichte, die über weite Strecken von dem Protagonisten selbst erzählt wird. Von der Warte der Narratologie gesehen lassen sich Schreiben und Suizid als erzähltechnische Probleme verstehen, die ihre Ereignishaftigkeit gemein haben. Ein Ereignis hat ein oder mehrere Zeitpunkte und ist mit ein oder mehreren Akteuren verbunden; es kann sich auch auf weitere Ereignisse beziehen. ${ }^{7}$ Gerade am Modellfall Briefroman mit seiner deutlichen und meist expliziten Datierung wird augenfällig, dass mit der Erzähleinheit des Briefes ein Schreib- oder Erzählereignis verbunden ist, welches zumindest einen Schreiber voraussetzt.

Der Einsatz ist bei einer Konstellation, in der jemand sich in der Fiktion ums Leben schreibt, einerseits Kunst (das Ergebnis des fiktionalen Schreibereignisses, der Roman Werther), andererseits das erzählte Leben (der Figur Werther). Hier kommt die autobiographische Subjektspaltung zum Tragen, die den im Ereignishorizont des Briefs Schreibenden vom vergangenen Ich des Schreibers scheidet. Eine solche Subjektspaltung charakterisiert auch den Suizid. So schreibt Théodore Jouffroy 1842, in einer Fußnote: "Suicide est un mot mal fait; ce qui tue n'est pas identique à ce qui est tué. « ${ }^{8}$ Die angesprochene Subjektspaltung geht im Falle Werthers, wo es um die literarische Schilderung suizidalen Verhaltens aus erster Hand geht, noch weiter: Der Getötete ist zugleich derjenige, der aufgeschrieben hat - der Tötende der Schreibende.

Eine der zentralen Innovationen des Briefromans als Gattung, die im 18. Jahrhundert eine rasche Karriere machte, besteht darin, dass die Erzählung sich immer aufs Neue von den Zeitpunkten der Niederschrift und des Abschickens zurück abwickelt, bis sich das Erzählte (mit Genette: die Geschichte) an den Ereignishorizont des Schreibens annähert. Das ist es, was Richardson mit »writing to the moment« meint, es wird nicht ex post berichtet, sondern mit jedem Briefeinsatz gibt es einen neuen Einsatzpunkt, von dem aus zurück erzählt wird. Der Schreibende kann niemals das Subjekt des Niedergeschriebenen sein, wer das eigene Leben berichtet, ist immer schon ein anderer

7 Zur Datenmodellierung von Ereignissen in Bezug auf Akteure und Entitäten vgl. FOKKENS, Antske, BRAAKE, Serge ter, Ockleloen, Niels, u. a., BiographyNet: Extracting Relations Between People and Events: Aspekte, Bausteine, Normen und Standards für eine europäische Biographik, in: Europa baut auf Biographien, hg. v. BERNÁD, Ágoston Zénó Bernád, GRUBER Christine, KAISER, Maximilian, 1. Auflage, Wien 2017, S. 193-223, das Datenmodell des Personendaten-Repositoriums an der Berlin-Brandenburgischen Akademie der Wissenschaften (http://pdr.bbaw.de/, letzter Aufruf: 27. 10. 2018), auch CZEITSCHNER, Ulrike, KRAUTGARTNER, Barbara, Discursive Constructions of Culture: Semantic Modelling for Historical Travel Guides, 5 (Apr, 2017) No 4, 2017, S. 323-331.

8 JOUFFROY, Théodore, Nouveaux mélanges philosophiques par Theodore Jouffroy, hg. v. Jean Philibert Damiron, Paris 1842, 245 Anm. 1, bei Macho, 16 in Übersetzung »Suizid ist ein schlecht gewähltes Wort; wer tötet, ist niemals identisch mit dem, der getötet wird.« 
als der, der es erlebt hat. Das ist ein Gemeinplatz der Erzählforschung, ${ }^{9}$ und gerade der Briefroman wird immer wieder als Beispiel dafür genommen, wo sich die zeitliche Distanz des Erzählzeitpunkts zur Zeit der Geschichte, also des Erzählten, verringert, und ein erzählendes Ich vor dem Problem steht, mit dem Bericht der Ereignisse dort angekommen zu sein, wo sie zu Ende sind und es sie nur mehr aufschreiben kann. In manchen Briefromanen wird das auch explizit gemacht, aber zur spezifischen Qualität des Werther gehört, dass so etwas eben gerade nicht vorkommt - hier hat Goethe an seinen Vorbildern viel gelernt und einen quasiauthentischen Schreibstil für seine Figur darin gefunden, dass auch der zeitgenössische Leser die Gattung und ihre stereotypen Formulierungen nur implizit mitliest.

Bei Werther und in einer ganzen Reihe weiterer literarischer Texte in der Folge von Goethes Roman werden die erwähnten beiden Arten der Subjektspaltung enggeführt: Töten und schreiben.

\section{Lektürereflexe im Werther und die Gattung}

Macho hat darauf hingewiesen, dass die zeitgenössische Auseinandersetzung mit Goethes Roman mit den Argumenten der Diskussion um die Ansteckung geführt wurde; die einschneidende Erfahrung der Pestepidemien in der Frühen Neuzeit mündet in ein Ansteckungs-Dispositiv, das die Wahrnehmung nicht nur von Krankheiten, sondern auch von kulturellen Artefakten, von Gedanken usw. deutlich prägt.

Dieses Dispositiv führt dazu, dass man vor Ansteckung Angst hat, und das betrifft auch Texte: Die Leser könnten vom »Werther-Fieber« (eine Krankheitsmetapher!) so weit angesteckt werden, dass sie sich selbst umbringen - das ist einer der wesentlichen Begründungsstränge für das Verbot von Goethes Roman durch die Zensur an vielen Orten. Für das Leipziger Verbot des Werther war übrigens, so schreibt sich das in der Forschung fort, der Dekan der Theologischen Fakultät mitverantwortlich, der ein entsprechendes Schreiben an den Leipziger Stadtrat unterfertigte.

Pro Memoria an die Churf. Bücher Commission

Es wird hier ein Buch verkauft, welches den Titel führt, »Leiden des jungen Werthers « usw. Diese Schrift ist eine Apologie und Empfehlung des Selbst Mordes; und es ist auch um des Willen gefährlich, weil es in wiziger und einnehmender Schreib Art abgefaßt ist. [...]

Leipzig am 28. Jan. 1775.

D. Joh. August Ernesti, als d. Z. Decanus der theol. Facultät allhier. ${ }^{10}$

Genau dieser Dekan wird in Goethes Roman genannt und angegriffen - es handelt sich um Johann August Ernesti, der unter vielen anderen Verdiensten auch eine Homer-Aus-

9 Vgl. etwa GENETTE, Gérard, Die Erzählung, übers. v. Andreas Knop, 3., durchgesehene und korrigierte Aufl., München 2010 (UTB 8083), S. 124, 164 u.ö.

10 Zit. n. FLASCHKA, Horst, Goethes »Werther«, Werkkontextuelle Deskription und Analyse, München 1987, S. 281. 
gabe verantwortet hatte und bei dem Goethe an der Leipziger Universität »eifrig mehrere Kollegia gehört hatte « ${ }^{11}$ Wenn Werther also am 28. August schreibt:

Heut ist mein Geburtstag, und in aller Frühe empfang ich ein Päkgen von Alberten. [...] Es waren zwey Büchelgen in duodez dabey, der kleine Wetsteinische Homer, ein Büchelgen, nach dem ich so oft verlangt, um mich auf dem Spaziergange mit dem Ernestischen nicht zu schleppen. ${ }^{12}$

dann bietet er genug Angriffsfläche (etwa für einen Zensureingriff vonseiten des Genannten), denn Ernestis Homer-Ausgabe war eine zeitgenössische Standardausgabe, zweisprachig in immerhin fünf nicht sehr tragbaren Bänden. Ein Verlangen nach einer Taschenbuchausgabe kann als Affront gegen einen ordentlichen Büchermann gelesen werden, der im 18. Jh. mit großer Sorgfalt mehrsprachige kommentierte Ausgaben nach dem aktuellen Stand der Edition veranstaltet hat, noch dazu, wenn dieses Verlangen selbst in einen Roman im Kleinoktavformat eingepasst ist.

Die Geschichte der Lektüren des Protagonisten Werther ist schon oftmals erzählt worden, auch wie sich seine Vorliebe von Homer weg und zu Ossian hin gewendet hat, ist bekannt, dass das auch als Wechsel des Erzählmodells verstanden wurde, liegt auf der Hand wie die küchenpsychologische Überlegung, dass es eine Übertragung der Themen wie auch der Darstellungsweise von den Gegenständen der Lektüre auf die Bewusstseinsinhalte der Hauptfigur gegeben haben könnte. Das ist von Goethe im Sinne einer Ansteckung eingestreut, er motiviert damit zusätzlich die psychosoziale Verfassung Werthers: Zuerst helle und klare Antike, dann dunkle und andeutungsreiche Romantik.

Allerdings ist die literarische Figur Werther im Roman nicht allein mit ihrer Abhängigkeit von Literatur, sondern auch Lotte als Objekt von Werthers Begierden ist von ihren Lektüren bestimmt:

Eine der ersten Erzähldigressionen Werthers im Brief vom 16. Juni 1771, in dem die Exposition des gesamten Romans offengelegt wird (dieser Brief ist mengenmäßig nach der langen Ossian-Passage gegen Schluss der umfangreichste Einzelteil des gesamten Textes), bezieht sich auf die Lektüre Lottes, die berichtet, dass sie früher gerne Romane gelesen habe, aber ihr damaliges Versinken in der Lektüre nicht mehr verstünde. Zum Erzählzeitpunkt begründet Lotte ihre Qualitätsanforderung gegenüber Literatur mit einer quantitativen Bedingung:

Doch da ich so selten an ein Buch komme, so müssen sie auch recht nach meinem Geschmakke seyn. Und der Autor ist mir der liebste, in dem ich meine Welt wieder finde, bey dem's zugeht wie um mich, und dessen Geschichte mir doch so interessant so herzlich wird, als mein eigen häuslich Leben, das freilich kein Paradies, aber doch im Ganzen eine Quelle unsäglicher Glükseligkeit ist. ${ }^{13}$

11 HOUBEN, Heinrich Hubert, Der polizeiwidrige Goethe, Berlin 1932, S. 7.

12 GOETHE, Johann Wolfgang, Die Leiden des jungen Werthers, Leipzig 1774, S. 98, Brief vom 28. August.

13 ebd, S. 34. 
Der Mangel an Lektüre und an Zeit für Lektüre befördert erhöhte Qualitätsansprüche, und die erzählte Geschichte sollte doch eigentlich keine andere Geschichte sein als eine, die man schon kennt. Ein geordnetes Leben abseits von Hofintrigen, so die Idealvorstellung der von Werther erzählten Lotte, inkludiert, dass man lesen kann; ferner, dass man lesen kann, was man möchte; empfindsames bürgerliches Leben nimmt auch Lektüre in seine Politik auf. Lottes Wunsch nach Unmittelbarkeit und >durchsichtiger< Übereinstimmung Transparenz von Büchern mit dem Leben läuft seiner mehrfachen Vermittlung über erstens die erzählte Rede, zweitens den Brief Werthers und drittens den Romandruck von Die Leiden des jungen Werthers geradewegs entgegen, sein Ausdruck beinhaltet also die Markierung seiner tendenziellen Unerfüllbarkeit. Auch Lotte befindet sich - von einem Standpunkt außerhalb der Erzählung betrachtet - mit ihren Lektürevorbildern in Einklang, sie hat sich anstecken lassen von den tugendhaften Romanen, die sie einst gelesen hat - genannt ist in der Passage bekanntlich nur Goldsmiths Landpriester von Wakefield, mitgemeint ist aber ein ganzes Reservoir an Lektüren, die nach der Meinung zeitgenössischer »Kunstrichter« für junge Frauen empfehlenswert sind und die als Erziehungsprogramm wirken sollen, um gegen die Ansteckung durch >üble Romanenlektüre $\mathrm{zu}$ immunisieren. Vorauszusetzen ist, dass die Gattung Roman in der deutschsprachigen Literatur bis in das letzte Jahrhundertdrittel einen eher schweren Stand hat, mit der ersten Ausnahme von Wielands Agathon (1766/67), der dafür gesorgt hat, dass dem Gattungstableau ${ }^{14}$ Roman bei Blanckenburg ${ }^{15}$ auch aus literaturgeschichtlicher Perspektive erste Aufmerksamkeit zuteilwurde, was zur Nobilitierung der ehedem verschrienen Gattung beigetragen hat. Goethe kann das mit »Goldsmith« angetippte Paradigma der Romangattung 1774 aber bereits ohne es durchzugehen auslassen; an dieser Stelle steht die von der Leserin auszudeutende Anmerkung des Herausgebers:

„Man hat auch hier die Namen einiger vaterländischen Autoren ausgelassen. Wer Theil an Lottens Beyfall hatte, wird es gewiß an seinem Herzen fühlen, wenn er diese Stelle lesen sollte. Und sonst brauchts ja niemand zu wissen." 16

Im weiteren Erzählverlauf ruft Lottes Lektüre Werthers Beifall hervor, bietet damit den ersten Grund für die Liebe, die dann am Schluss des zitierten Briefs in den bekannteren »Klopstock! «-Ausruf münden und den Gleichklang zweier Herzen über ihre Lektüreerfahrungen so weit koppeln kann, dass durch die Unmöglichkeit der Liebe aus der Figurensicht Werthers nur mehr der Suizid als Möglichkeit bleibt. Die verhängnisvolle Ereignis- und Lektürenkette wird zuerst durch Lottes Lektüre (freilich vermittelt über die Erzählung des Protagonisten) angestoßen.

Der Einschluss in eine Gattung funktioniert über Lektüren, und zwar bereits innerhalb der Erzählung, das demonstrieren der Briefschreiber Werther im Dialog mit der nur aus seiner Sicht dargestellten Lotte und unter Einmischung des Herausgebers, der lauschend mitliest und die extradiegetische Kommunikation zwischen dem Roman und

14 PLUMPE, Gerhard, Ästhetische Kommunikation der Moderne, I, S. 199.

15 BLACKENBURG, Friedrich von, Versuch über den Roman, Leipzig; Liegnitz 1774.

16 GOETHE, S. 35. 
dem Leser in der Fußzeile bricht. An der Entstehung und Konsolidierung einer Gattung sind immer mehrere Akteure und Agenturen beteiligt ${ }^{17}$ das beginnt

1. erstens bei den Autoren, die ihre Texte anhand von Vorbildern (Architexten, Prätexten), expliziten Gattungsbezeichnungen im Paratext ${ }^{18}$ und sim Wege der Intertextualität $<$ in eine Reihe von weiteren Texten einfügen.

Beim Werther ist es klar, dass Johann Wolfgang Goethe die Gattung intensiv studiert hatte und dann anlässlich seiner Kestner-/Buff-Erlebnisse sowie mit dem Modell von Jerusalems Suizid entsprechend anwenden konnte. Für Goethe ist der Werther der einzige Briefroman geblieben, die Gattung hat er verlassen, gleichzeitig aber mitgeprägt, da der Text mit dem Suizidthema neben der aufkommenden Modegattung auch noch einen zweiten Nerv der Zeit getroffen hat.

Wo die Vorbilder liegen, daran kann kein Zweifel bestehen; Goethe schreibt am 24.6.1783 an Charlotte von Stein anlässlich seiner Lektüre des Werther in englischer Übersetzung: »Mir war's gar anmuthig meine Gedancken in der Sprache meiner Lehrer zu lesen. « ${ }^{19}$ Goethe ist erst Leser und seine »Lehrer « sind andere Autoren.

2. Zweitens sind beim Gattungsprozess die Agenturen der Distribution beteiligt (also Verlage, Messen, Buchhandlungen, Bibliotheken), welche über Paratexte, Katalog-Kategorisierung, öffentliche Epitexte die Gattungszugehörigkeit eines von ihnen verteilten Textes bestätigen, unterlaufen, überschreiben oder erstmals fixieren können.

Der Briefroman hat dabei eine bestimmte optische Form, die man mit Susanne Wehde als »typographisches Dispositiv «, ${ }^{20}$ als gattungsprägendes Superzeichen verstehen kann; diese geht interessanterweise auf die Vorbilder gedruckter realer Briefwechsel zurück, sodass man hier - mit Bachtins Essay über die Sprechgattungen $^{21}$ - von einer sekundären Verwendung einer anderen Gattung sprechen kann. Im konkreten Fall ist kein Einfluss Goethes auf den Druck und auf die Mechaniken des Buchdrucks nachzuweisen, im Gegenteil: Der Leipziger Verleger Weygand, bei dem die Erstausgabe 1774 erschien, war ein Verleger, der auf Massenproduktion setzte; ${ }^{22}$ wie bekannt hat er nicht nur von Goethes Roman hinter dem Rücken

17 Zum Folgenden vgl. MICHLER, Werner, Kulturen der Gattung. Poetik im Kontext, 1750-1950, Tübingen 2015.

18 GENETTE, Gérard, Paratexte. Das Buch vom Beiwerk des Buches, Frankfurt am Main 2001 (stw 1510).

19 Goethe, Bd. 6 Briefe, Tagebücher, Gespräche, S. 175.

20 WEHDE, Susanne, Typographische Kultur. Eine zeichentheoretische und kulturgeschichtliche Studie zur Typographie und ihrer Entwicklung, Tübingen 2000 (Studien und Texte zur Sozialgeschichte der Literatur 69), S. 119.

21 BACHTIN, Michail M., Sprechgattungen, hg. v. Rainer Grübel, Renate Lachmann, Sylvia Sasse, übers. v. Alfred Sproede, Rainer Grübel, Berlin 2017.

$22 \mathrm{Zu}$ Weygand vgl. KOCH, Herbert, WEYGAND, Johann Friedrich, Buchhändler in Leipzig, in: Archiv für 
seiner Autoren Titelauflagen veranstaltet; da kann sich so ein Verleger nicht dreinreden lassen, sondern er lässt nach dem gültig-erwartbaren Schema absetzen.

Die Zensur gehört zu den institutionellen Akteuren der Distribution bzw. ihrer Verhinderung; um die Gattung geht es den Zensoren im konkreten Fall aber nicht, sondern nur um die besondere Verführungskraft von Goethes Roman aufgrund seines Stils, »weil es in wiziger und einnehmender Schreib Art abgefaßt ist."

3. Drittens nehmen Leserinnen und Leser anhand von Ähnlichkeitsrelationen aus ihrer bisherigen Lektüre, Vorbildung, Schulkategorien und Kanon gattungshafte Zuordnungen vor, die Texte ebenfalls in Reihen oder Gruppen zusammengefasst verstehbar machen. Als Briefroman ist Goethes Text von den Viellesern sicher schnell erkannt worden, aber zumindest teilweise auch als Lebensdokument, was in der dokumentarischen Authentizitätsfiktion der Gattung als Negation des Übergangs von der primären Gattung Brief in die sekundäre Gattung Briefroman bereits angelegt ist.

4. Eine vierte Agentur kommt ab ca. der zweiten Hälfte des 18. Jahrhunderts mit der professionellen Lektüre hinzu, zu Werthers Zeit hießen diese »Kunstrichter«, gemeint ist also die Literaturkritik, später dann auch die Literaturwissenschaft die im Fall der Neugermanistik von ihrer Genese her zuerst eine Goethe-Philologie war. ${ }^{23}$

In deren Gründungsopus, der Sophienausgabe, fällt der Werther, herausgegeben von dem Grazer Extraordinarius Bernhard Seuffert und Konsorten, allerdings durch die Verpflichtung auf die Gesamtanlage der auf 143 Bände angelegten Ausgabe methodisch bereits vor dem Erscheinen hinter den zeitgenössischen Forschungsstand zurück.

Seuffert beklagt sich im jüngst edierten Briefwechsel mit August Sauer 1897 und 1898 über das langsame Fortgehen der Werther-Edition; »Jetzt bereite ich colleg vor und dann gehe ich an den Werther! mir graust vor der Collationirerei! wenn nur was herauskäme! «. ${ }^{24}$ Daraus spricht der hohe und industrialisierte Stand des Verlagswesens im letzten Drittel des 18. Jahrhundert, der zu geringer Varianz geführt

\footnotetext{
Geschichte des Buchwesens, 9, 1969, 434-448, BRANDSCH, Juliane, HENTSCHEL, Uwe, REK, KLAUS, u. a., Das literarische Leipzig. Kulturhistorisches Mosaik einer Buchstadt, hg. v. Andreas Herzog, Leipzig 1995, Reinhard Wittmann, Weygand - der »Fabrikbuchhändler«, in: Das literarische Leipzig. Kulturhistorisches Mosaik einer Buchstadt, hg. v. HERZOG, Andreas, Leipzig 1995, S. 126-127.

23 Vgl. MICHLER, Werner, An den Siegeswagen gefesselt. Wissenschaft und Nation bei Wilhelm Scherer, in: Literatur und Nation. Die Gründung des Deutschen Reiches 1871 in der deutschsprachigen Literatur, hg. v. AMANN, Klaus, WAGNER, Karl, Wien; Köln; Weimar 1996 (Literatur in der Geschichte, Geschichte in der Literatur 36), S. 233-266.

24 Seuffert, Bernhard an August Sauer, Brief 5. Oktober 1897. In: Briefwechsel Sauer-Seuffert (online) http://sauer-seuffert.onb.ac.at/node/8865, letzter Aufruf 27.10.2018.
} 
hatte und der auch die methodischen Vorgaben der frühen Neugermanistik auf die Probe stellte. ${ }^{25}$

Gattung entsteht multifaktoriell, sie ist keinesfalls lediglich von thematischen, motivischen oder stofflichen Faktoren abhängig; beim Briefroman sind aber auch noch einfach zu erkennende formale Eigenschaften als Paradigma von Merkmalen wirksam (ähnliches gilt für das Sonett, es hat immer 14 Verse).

Der Werther-Stoff wandert, wobei das Suizidmotiv unterschiedliche Ausprägungen und Abwandlungen erfährt, von denen einige Beispiele zeugen.

\section{Abwandlungen zum Suizidmotiv}

Weg vom Werther: Der Suizid ist nicht die einzige Lösung, um die Protagonisten und Protagonistinnen empfindsamer Romane zu entsorgen. Zeitgenössisch sind es eher Auswege wie die Heirat im Fall des happy endings, oder für Frauen, gefallene Mädchen oder sonst dem Suizid entgangene Weibspersonen die Alternative, ins Kloster zu gehen; das ist seinerseits topisch geworden und hat zu Klosterromanen geführt, in denen die Ausschmückung der Ausschweifung mitunter auch nicht jugendfrei im Sinn des frühen 19. Jahrhunderts erscheint.

Für männliche Hauptfiguren (hier besteht eine deutliche Genderdifferenz dahingehend, wie erzählerisch mit Frauen und wie mit Männern umgegangen wird) jedoch ist auch eine andere Form von Gewaltakt, nämlich Homizid, Mord oder Totschlag, ein veritables Gefühlsventil. Noch eine Variante gibt es, und zwar die Selbstopferung des Märtyrers, so wie das am Ende des dritten Bandes von Caroline Pichlers Agathokles auftritt: Hier steigt der im Rahmen der Christenverfolgung gefangengenommene Titelheld auf das »Todesgerüst, bethete mit stiller Rührung - und so verließ der Schatten des edelsten Mannes die Erde «. ${ }^{26}$

Neben dem Martyrium liegt im literarisch-motivischen Nahebereich des Suizids auch der Mord, der als literarisches Motiv insgesamt gesehen häufiger vorkommt jenes der Selbstopferung. ${ }^{27}$ Betreffend den aus dem Suizidwunsch (einer Werther-Figur)

25 Wie viel an Varianz Seuffert in der Überlieferungsgeschichte zu den Handschriftfragmenten und Drucken zum Werther trotz seiner Nullmeldung an Sauer herausarbeiten konnte, lässt sich einerseits anhand des Apparats feststellen, andererseits in Bernhard Seuffert, Philologische Betrachtungen im Anschluß an Goethes Werther, in: Euphorion, 7, 1900, 1-47; dort mit der methodischen Aufforderung verknüpft, die Überlieferung von gedruckten neuzeitlichen Texten analog zu jenen des Mittelalters auf der Suche nach dem »echte[n] Wort « ernst zu nehmen .

26 PICHLER, Caroline, Agathokles, Wien 1808, III, 310. Zur theologischen Abgrenzung zwischen Suizid und Märtyrertum verweise ich auf die einschlägigen Stellen bei Macho, v.a. S. 67-73.

27 Die Vermutung lässt sich tentativ bestätigen anhand der ngrams-Suche sowohl durch das englischsprachige als auch das deutschsprachige Korpus von Google Books, wo - mit Ausnahme der Jahre 1904-1911 im deutschsprachigen Korpus - die Häufigkeitskurven stets auf ein Überwiegen von murder/Mord gegenüber suicide/Selbstmord hindeuten. Alternative Suchbegriffe (mord,suizid,selbstmord,selbsttötung,tötung) liegen weit unter den genannten häufigeren. Vgl. https://books.google.com/ngrams. 
entwickelten homizidalen Übertretungen folgt an dieser Stelle ein Beispiel aus 1780, das in der Forschung nur als Sonderfall einer Wertheriade erwähnt wird:

\section{Vom Suizid zum Homizid: Herbert Croft, Love and Madness}

Der Roman Love and Madness stammt von Herbert Croft. ${ }^{28}$ Ein gewichtiges Indiz dafür diesen Text als Wertheriade zu lesen, besteht darin, dass der dortige Protagonist den Werther gelesen hat und darüber auch brieflich mit seiner Geliebten kommuniziert: Sie hat ihm nahegelegt, den Werther gerade nicht zu lesen, ein Verbot, das dieser folgenreich missachtet.

Der Plot ist deutlich in Parallele zum Vorbild Werther gebaut; die Briefdaten sind in dem Diagram eingetragen, um die äußere Struktur der beiden Texte nebeneinanderzustellen. Die Skalen sind notwendigerweise angepasst, weil Crofts Roman einen längeren Zeitraum erzählter Zeit abdeckt als Goethes Werther. In der Projektion sieht man die Ähnlichkeiten der chronologischen Konstruktion der beiden Romane in Relation zueinander: Häufungen zu Beginn und gegen Ende der Romane; mit dem ausführlichen Chatterton-Einschub und den Ossian-Zitaten wird gegen Schluss beider Romane sogar ein ähnliches retardierendes Element gesetzt.

Der Suizid schlägt in Crofts Roman allerdings fehl: Mr. H-, so heißt der Protagonist, erschießt sich nicht selbst, sondern er richtet die Geliebte, nachdem er sie mit einem anderen im Theater gesehen hat. Dabei verwendet Mr. H- allerdings keine landläufige Kugel, sondern er lädt seine Pistole mit einem Liebesbrief der Geliebten, die damit über die Bande doch von eigener Hand bzw. Handschrift stirbt. Danach versucht er sich selbst zu töten, aber wegen Ladehemmung missglückt der Suizid, wogegen der Homizid erfolgreich ist.

Mr. H- wird hingerichtet, nachdem er im Gefängnis auf die Urteilsvollstreckung wartend die letzten Briefe des Romans - darunter eine Abhandlung über den literarischen Schwindler Thomas Chatterton (über 120 Seiten!) ${ }^{29}$ - geschrieben hat. Im Roman kursiv gedruckt ist ein Hinweis einer nicht spezifizierten Erzählstimme, die als Drucker und/ oder Herausgeber, jedenfalls als Akteur der Distribution auftritt, und die nach dem Tod des Protagonisten am Galgen die Auffindungs- und Veröffentlichungsgeschichte zu den Briefen wiedergibt (sie argumentiert in Analogie zu Goethes zweiter Werther-Fassung von 1787 mit dem abschreckenden Beispiel); diese Passagen geraten hier anders als im Werther mit seinem ausgeprägten »Herausgeberteil« (von welchem Croft allerdings die Nichtdatierung der letzten Briefe Mr. H-s aus der Kerkerzelle übernimmt) zur bloßen Rahmung.

28 CROFT, Herbert, Love and Madness. A story too True in a Series of Letters Between Parties, whose Names would perhaps be mentioned, were they less known, or less lamented, London 1780.

29 Für diese Passagen hat Croft der Schwester des romantischen Paradesuizidenten unter Vorspiegelung falscher Tatsachen Dokumente entlockt, um diese im Roman ohne Erlaubnis zu publizieren, vgl. die Angaben zu Croft in der Encyclopaedia Britannica 1911, VII, 480. 
Dass Love and Madness einen realen Kriminalfall aufnimmt, der sich 1779 in London ereignete, hat dazu geführt, dass der Roman nicht als Wertheriade verstanden wurde, obwohl er zumindest in der Chatterton-Forschung nicht gänzlich vergessen ist. In der Werther-Forschung hat Georg Jäger auf einen Text von Willibald Alexis (d.i. Georg Wilhelm Heinrich Häring) hingewiesen, der unter dem Titel Ein englischer Werther 1843 in der Leipziger Penelope erschien. ${ }^{30}$ Der letzte Satz stellt die Verbindung zu Crofts Roman her:

Oft ist James Hackmans Leben und seiner Margaret in England beschrieben worden. Der Geistliche Dr. Herbert Croft hat zuerst ihren Briefwechsel gesammelt und herausgegeben. ${ }^{31}$

Bei genauerem Hinsehen erweist sich Alexis' knapp 70 Seiten umfassende Zeitschriftenerzählung als kürzendes und entstellendes Übersetzungsplagiat mit einer einleitenden Ich-Erzählung der Auffindung durch den Erzähler auf den ersten fünf Druckseiten. Bereits durch die Titelwahl ist der Bezug zu Goethes Text hier verdeutlicht, was auf publikationstaktische Gründe zurückzuführen sein mag; die Referenzen auf den realen Kriminalfall um Martha Ray und James Hackman, der 1779 am Tyburn hingerichtet wurde, sind in der Rahmenerzählung zusammengenommen und für das Zielpublikum ausgeschmückt, das anders als das Londoner Publikum Crofts 1780 zeitlich und räumlich zu weit entfernt gewesen wäre, um die Skandalgeschichte zu kennen. Der Chatterton-Plot des Croft'schen Textes fällt Alexis' Paraphrasen zum Opfer. Die deutsche und die englische Rezeption der Texte von Alexis und Croft laufen einerseits hinsichtlich der medientechnischen Voraussetzungen, andererseits hinsichtlich der gattungstechnischen Umsetzung - bei Croft der Briefroman, bei Alexis eine Erzählung mit längeren Passagen in Briefen, die durch paraphrasierende Übersprünge den weitgehend an Crofts Briefreihenfolge sich haltenden Abschnitten folgen - in erstaunlichem Maße auseinander. Ein englischer Werther ist nicht eine späte und untypische Wertheriade von $1843,{ }^{32}$ sondern nur die gekürzte Version eines der ersten Texte in Romanform, in denen der Goethesche Text in Form eines Reimports der Gattung Briefroman bereits 1780 »in der Sprache meiner Lehrer« (Goethes) wirkmächtig wird, und ist damit eine sehr frühe englische Wertheriade.

Zwei vom Werther deutlich abgegrenzte Motivvariationen zum literarischen Suizid nach einem Sprung von etwa 120 Jahren - »Fin-de-Siècle-Suizide « ${ }^{33}$ - ergänzen zur Illustration die Spannbreite der Reihe stofflich-thematischer >Ansteckung \ mit je unterschiedlichem Werther-Bezug.

30 Vgl. JÄGER, Georg, Die Leiden des alten und neuen Werther. Kommentare, Abbildungen, Materialien zu Goethes Leiden des jungen Werthers und Plenzdorfs Neuen Leiden des jungen W., München; Wien 1984 (Literatur - Kommentare 21), S. 11-45.

31 ALEXIS, Willibald, Ein englischer Werther, in: Penelope. Taschenbuch auf das Jahr 1843, Leipzig 1843, S. 1-69, hier S. 69.

32 JÄGER, S. 44.

33 So der Titel eines Abschnitts bei Macho, S. 106. 


\section{Vom Suizid zum Duell: Arthur Schnitzler, Lieutnant Gustl}

In Arthur Schnitzlers Monolognovelle Lieutnant Gustl ist der Suizid bereits fest beschlossen und die Abschiedsbriefe sind zumindest skizziert, als den Protagonisten die Nachricht erreicht, dass sich sein Ehrenproblem mitsamt seinem Beleidiger, dem Bäckermeister Habetswallner, nach dessen tödlichem Schlaganfall in Nichts aufgelöst hat. Damit kann der Suizid abgesagt werden und Gustl sich seiner nächsten möglichen Todesursache zuwenden. Es ist eine fröhliche literarische Apokalypse, die in der Darstellung des moralischen Zustandes der Offiziere der K.u.K. Armee - gezeichnet durch den inneren Monolog dieses »dummen Buben« Gustl - die außerliterarische Folge der Degradierung des Autors Schnitzler zeitigte. ${ }^{34}$

Schnitzlers Innovation des Inneren Monologs erscheint mit ihrer radikalen Subjektivität als logische Konsequenz und Weiterentwicklung des Briefromans. ${ }^{35}$

Obwohl sich Schnitzlers Erzählung vordergründig mit dem durch Verletzung an der Ehre bedingten geplanten Suizid befasst, gibt es eine zweite Schicht, die jenseits der erzählten Handlung liegt. Es handelt sich bei der zweiten Motivkonstellation um das bevorstehende Säbelduell Gustls mit dem mehrfach genannten »Doktor«: Einerseits lässt sich das Duell als ritualisierte wechselseitige Suizidhandlung auffassen - ein weiteres literarisches Beispiel zeitlich näher am Werther wäre das Schlusstableau von Ludwig Tiecks William Lovell, wo sich der schwärmerische Held, nachdem er erkannt hat, dass er zum Spielball des Bösen geworden ist, im mit Pistolen ausgeführten Duell eine Malvenblüte als Zielscheibe an die Brust heftet und selbst nicht schießt. ${ }^{36}$

Andererseits ist mit dem letzten Satz von Schnitzlers Lieutnant Gustl, »Dich hau' ich zu Krenfleisch! «37 in zweierlei Hinsicht der Suizid zum Homizid hin gedreht: Erstens ist Gustl überzeugt davon, den Doktor - er ist Jurist und nach der Mutmaßung Gustls Sozialist - im Duell zu besiegen, wenn nicht gleich zu töten; zweitens und dies verstärkend ist auch das Manuskript an dieser Stelle selbst für Schnitzlers bekanntlich ärztliche Handschriftenverhältnisse expressiv: ${ }^{38}$ Die auf die letzte Manuskriptseite geworfenen Schlussworte »zu Krenfleisch!« deuten (was in den gedruckten Quellen zum Text wieder

34 Aus der umfassenden öffentlichen Diskussion, die der Veröffentlichung der Novelle in der Weihnachtsausgabe der Neuen Freien Presse vom 25.12.1900 folgte, sei der Schlusssatz des Leitartikels der Reichspost vom 22.6.1901, 1 herausgegriffen, da er die Leitbegriffe dieser Diskussion griffig zusammenfasst: »Vor allem reinige das Officierscorps selbst seinen Officiers-Ehrenbegriff, dann räume man mit Gesetzen, Traditionen und Moden auf, die zum Duell zwingen, die zum Dreinhauen mit der Waffe gegen Wehrlose zwingen und die eventuell sogar zum Selbstmord treiben.« (Hervorhebung im Original durch Sperrdruck)

35 Die Verbindung dieser beiden Texte miteinander hat, soweit ich sehe, zuerst vorgeschlagen: KARTHAUS, Ulrich, Zweihundert Jahre »Werther«, in: Gießener Universitätsblätter, 2, 1975, S. 61-82, hier S. 75; vgl. auch A companion to the works of Arthur Schnitzler, hg. v. LORENZ, Dagmar C. G., Rochester, NY u.a. 2003 (Camden House companion volumes), 134 f. Schnitzler sah im Februar 1892 die Uraufführung von Massenets Werther in der Wiener Hofoper, am 28. September 1920 notiert er »Begann, ,Werther zu lesen.« vgl. SCHNITZLER, Arthur, Tagebuch: 1879-1931, hg. v. Werner Welzig, Peter Michael Braunwarth, Wien.

36 TIECK, Ludwig, William Lovell, Berlin; Leipzig 1795/96, III, S. 474.

37 SCHNITZLER, Arthur, Lieutnant Gustl, in: Neue Freie Presse, 13053, 1900, S. 34-41, hier S. 41.

38 Vgl. die Reproduktion in der historisch-kritischen Schnitzler-Ausgabe, Arthur Schnitzler, Werke in historisch-kritischen Ausgaben, hg. v. FLIEDL, Konstanze, Bd. 1, Berlin 2011, Lieutnant Gustl, S. 508 [H240]. 
in die normierten Zeichenbestände der Druckschrift nivelliert ist) nicht nur auf einen vom Protagonisten vorhergesehenen, sondern auch vom Autor vorgesehenen Tod des studierten Militärverächters hin, was aber nicht einmal einem Graphologen vom Schlage eines Ludwig Klages zustünde zu behaupten. Ganz unbegründet kann der Verdacht aber nicht sein, vorsichtiger wurde für das gesamte Manuskript Schnitzlers formuliert:

In der Handschrift kommt es dadurch [durch die durchgängige Verwendung des »Inneren Monologs«] zu einer bemerkenswerten Überlagerung von produktionsästhetischer Assoziation und figuralem Bewusstseinsstrom, von Schnitzlers Schriftflüchtigkeit und Gustls Gedankenflucht. [...] In seinem affektiven und expressiven Schreibduktus ist das Gustl-Manuskript der Schrift gewordene Innere Monolog. ${ }^{39}$

Wie das außerhalb des Erzählhorizonts liegende Duell Gustls mit dem Doktor ausgeht, ist angesichts der Tatsache, dass man Schnitzlers Protagonisten nie so ganz trauen kann - wie sich das Fräulein Else genau an die Letaldosis des barbiturathaltigen Präparats Veronal hält, ${ }^{40}$ sodass auf der extradiegetischen Ebene ein offenes Ende übrigbleibt - reichlich unklar. Der Suizid des Tötungshandwerkers, so viel steht fest, findet in Schnitzlers Novelle nicht statt, an seine Stelle schiebt sich die ritualisierte Tötungshandlung Duell - erzähltechnisch durch den Effekt der Fokalisierung auf den Ich-Erzähler Gustl bekanntermaßen in groteske Abschreckung gekippt.

\section{Vom Doppelsuizid zum Mord: Heinrich Mann, Pippo Spano}

Drei Jahre nach dem von einem Literaturskandal gefolgten Erscheinen von Schnitzlers Lieutnant Gust $l^{41}$ schreibt Heinrich Mann seine Novelle Pippo Spano, in der das Suizidmotiv eine weitere Abwandlung erfährt. In diesem Text mündet die idyllische Geheimliebe zwischen dem Schriftsteller Mario Malvolto und der jungen Adeligen Gemma Cantoggi in eine Katastrophe, als die beiden nackt auf der Terrasse von Malvoltos Haus fotografiert werden ${ }^{42}$ Kunstvoll ist über den ganzen Text hinweg angedeutet, dass nach dieser intensiven Liebe nur mehr der Tod kommen können wird - und als der Skandal unausweichlich scheint, nehmen sich beide vor, gemeinsam ihrem Leben ein Ende zu setzen.

39 ebd, 3 [Editorische Hinweise], zum Formalen vgl. auch Achim Nuber, Neue Aspekte zu Arthur Schnitzlers Monolognovellen »Leutnant Gustl« und »Fräulein Else«, in: Zeitenwende - die Germanistik auf dem Weg vom 20. ins 21. Jahrhundert, Akten des X. Internationalen Germanistenkongresses Wien 2000. Epochenbegriffe: Grenzen und Möglichkeiten; Aufklärung - Klassik - Romantik; Die Wiener Moderne, hg. v. WIESINGER, Peter, JAPP, Uwe, MAEDA, Ryozo, u. a., Bern u.a. 2002, S. 427-432, S. 428 f.

40 Dies hat Verf. in anderem Zusammenhang nachgewiesen, vgl. »A. ist manchmal wie ein kleines Kind «. Clara Katharina Pollaczek und Arthur Schnitzler gehen ins Kino, hg. v. Verf., ROHRWASSER, Michael, SCHOPPER, Daniel, Wien 2012 (manu scripta 2). S. 31, Anm. 67.

41 Umfassend dokumentiert basierend auf Schnitzlers Zeitungsausschnittsammlung und den Egodokumenten etwa aus den Tagebüchern ist dies in RENNER, Ursula, Dokumentation eines Skandals: Arthur Schnitzlers »Lieutenant Gustl«, in: Hofmannsthal-Jahrbuch zur europäischen Moderne, 15, 2007, S. 33-216.

42 Vgl. BEZ, Martin, »... man hat uns photographiert«: Anmerkungen zu Heinrich Manns Novelle »Pippo Spano«, in: Heinrich-Mann-Jahrbuch, 30, 2012, S. 129-146. 
Bevor die in einer Männerphantasie dem Dichter Malvolto verfallene Cantoggi ihre Affäre bekommt (sie ist verlobt mit Lanti, »ein[em] Viveur auf dem Abmarsch «), ${ }^{43}$ wehrt der Verführte noch mit einer Begründung ab, die dem Goetheschen Albert eingefallen sein könnte: »Glaube, dein Verlobter, er mag kalt sein - er hat immer noch mehr gutes Gefühl als ich. Er ist dir immer noch verwandter. ${ }^{44}$ Der Vorschlag zum Doppelsuizid kommt in direkter Reaktion auf die erste gemeinsame Liebesnacht ursprünglich von Malvolto, in einem nicht abgeschickten Brief, den er vernichtet:

All meine Sehnsucht drängt nach den Starken, die das könnten, nach den Condottieri des Lebens, die in einer einzigen Stunde ihr ganzes Leben verschlingen und glücklich sterben. Anstatt uns nun trübe zu verlassen, hätten wir heute früh zusammen sterben sollen, o Gemma! ${ }^{45}$

Der Clou des letzten Kapitels Die Tat besteht darin, dass als Handlungsmotivation dafür, den Versuch diese Selbstmordphantasie ins Werk zu setzen, ausgerechnet ein Medienereignis herhalten muss - die fotografische Ablichtung des nackten Paares auf der Terrasse. Malvolto ruft anlässlich der Neuigkeit: »Unerhört! Das ist unerhört. Da ich mich doch vergewissert habe, daß von keinem Punkt der ganzen Umgebung meine Terrasse zu entdecken ist! Es muß vom Garten aus geschehen sein. ${ }^{46}$ Dies führt zur Befürchtung öffentlichen Ehrverlustes für beide und mittelbar zum gemeinsamen Todesbeschluss. Davor steht allerdings noch die Drohung des Duells mit dem Bruder der Gemma, einem Soldaten. Gemma weiß gegen ein solches einzuwenden:

»Tötest du ihn, sind andere Verwandte da, und sie werden uns trennen. Ich bin erst siebzehn. [...] Siehst du, dann müßten wir dennoch sterben. Warum wolltest du vorher meinen armen Bruder töten? Sterben wir lieber gleich jetzt. « ${ }^{47}$

Dass Gemma Cantoggi unmittelbar darauf als »kindliche Judith« bezeichnet wird, passt in das Bild des ästhetizistischen Orientalismus (der in Manns Text auch sonst ausführlich bedient wird).$^{48}$

Malvolto, der sich so stark zu sein wünscht wie der titelgebende Türkenbezwinger aus der Renaissance (Philippe Scolari), ${ }^{49}$ schafft es gerade, der Geliebten den Dolch hineinzustoßen; bei sich selbst verzagt er - er hat sich nur geritzt; sie merkt das sterbend und

43 MANN, Heinrich, Künstlernovellen: Pippo Spano, Schauspielerin, Die Branzilla, Stuttgart 1987 (Universal-Bibliothek 8381), S. 12.

44 ebd, S. 21.

45 ebd, S. 27.

46 ebd, S. 27.

47 ebd, S. 43.

48 Zum Darstellungstypus Judith u.a. vgl. LUDEWIG, Anna-Dorothea, »Braut des hohen Liedes «: ’Jüdinnenbilder im Werk von Leopold von Sacher-Masoch, in: Zagreber germanistische Beiträge, 26, 2017, S. 233-252.

49 Vgl. Peter RIEDL, Peter Philipp, Renaissancemenschen: Literarische Konstruktionen eines Figurentyps um 1900, in: Der Renaissancismus-Diskurs um 1900, Bielefeld 2017, S. 71-97. 
beschimpft ihn als Mörder. »Was geht das Geschick dieser Sterbenden mich an!«, sagt er noch. ${ }^{50}$

Bei Heinrich Mann ist es ein gefeierter Schriftsteller, der sich nicht umbringt - im Unterschied zu Die Leiden des jungen Werthers, worin sich der Schreiber tötet, der eigentlich Maler ist und doch nichts herausbringt als Brieftext, auch zu Love and Madness, worin der Briefschreiber die Geliebte tötet (ein Soldat, der sich zum Priester weihen hat lassen), auch zu Schnitzlers Gustl, worin sich der Ehrbeleidigte nicht tötet, um um 4 Uhr nachmittags präsumtiv jemanden anderen zu töten. Dennoch ist in allen vier hier kurz behandelten Fällen der unmittelbare Bezug zwischen dem Schreiben und dem Töten vorhanden, der in der Subjektspaltung mündet; nur ist der Persönlichkeitsanteil des Tötenden auf das Gemälde des Pippo Spano projiziert, auf den Starken, der es fertigbrächte, sich zu töten.

\section{Erfolgreicher Schluss: Jakob Julius David, Ein Poet?}

Literarische Texte müssen sich zu kanonischen Prätexten wie Goethes Werther-Roman `verhalten`, andererseits erfordern aber die Bedingungen jeder fiktionalen Welt eigene strukturelle und narrative Konstruktionen. Beides gilt besonders in Hinblick auf die forcierte Engführung von Schriftverkehr und Gefühlsregelung: suizidale Schriften und schriftliche Suizide.

Zusammenfassen lässt sich die Verbindung von Subjektspaltung, Ansteckung, Motivvariation beispielhaft anhand eines weiteren Suizid-Textes, der gleichwohl mit dem Schreiben eng zusammenhängt und dessen Protagonist einen Gegenpol auch zu Schnitzlers dummem Leutnant Gustl einnimmt. Ich komme noch einmal auf die Frage von Leben bzw. Sterben und Schreiben zurück, die im ersten Kapitel zentral war:

In Jakob Julius Davids ${ }^{51}$ Novelle Ein Poet?, zuerst erschienen ab dem 9. Dezember 1892 in vier Fortsetzungen in der Wiener Arbeiter-Zeitung, ${ }^{52}$ geht es um einen mittellosen Zeitungsschreiber, um das akademische Proletariat der Wiener Jahrhundertwende. Dieser Josef Bernhofer kann schreiben, aber nachdem seine Texte zu empfindsam stimmungsvoll

50 Zum »verbrecherischen Lustmord « wird die Selbstmordschwäche Malvoltos bei THUMS, Barbara, Die Macht der Bilder: oder der Kampf ums Dasein in Heinrich Manns Künstlernovelle »Pippo Spano«, in: Heinrich-Mann-Jahrbuch, 24, 2006, S. 45-60, hier S. 45 im Zusammenhang mit den Devianzphänomenen der Dekadenz (Stichwortgeber sind Nietzsche und Krafft-Ebing).

51 Zu David biografisch vgl. ÖBL 1815-1950, Bd. 1, 171; Vancsa, Kurt: David, Jakob Julius, In: Neue Deutsche Biographie 3 (1957), 536; https://limam.upol.cz/Authors/Detail/62, letzter Aufruf: 27.10.2018; einen Überblick über die spärliche deutschsprachige germanistische Forschungsliteratur bietet: Clemens Peck, »Paralysis progressiva «. Zur Figuration des Bildungsproletariats in Jakob Julius Davids Wien-Roman Am Wege sterben, in: IASL, 2, 2010, S. 37-60.

52 1. 9. 12. 1892,10 http://anno.onb.ac.at/cgi-content/anno?aid=aze\&datum=18921209\&seite=10; 2.23. 12. 1892, $10 \mathrm{http://anno.onb.ac.at/cgi-content/anno?aid=aze \& datum=18921223 \& seite=10;} \mathrm{3.} \mathrm{30.} \mathrm{12.} \mathrm{1892,}$ 6 http://anno.onb.ac.at/cgi-content/anno?aid=aze\&datum=18921230\&seite=6; 4. 6. 1. 1893, 9 http://anno. onb.ac.at/cgi-content/anno?aid=aze\&datum=18930106\&seite=9, letzter Aufruf: 27. 10. 2018; Vgl. Jakob Julius David, Ein Poet?, in: Gesammelte Werke, hg. v. HEILBORN, Ernst, SCHMIDT, Erich, Bd. 3, München; Leipzig 1908, S. 152-191. 
sind, stets abschweifen und wesentliche Fakten auslassen, schreibt er oft umsonst und keine Redaktion übernimmt seine Berichte. Sein letzter Text lautet:

»(Selbstmord.) Heute morgen wurde im Prater nächst der Krieau der Leichnam eines etwa vierzigjährigen Mannes gefunden. Der Unglückliche, der sich durch einen Revolverschuß in die rechte Schläfe getötet hatte, wurde durch die bei ihm vorgefundenen Papiere als der Dr. phil. Josef Bernhofer, der zuletzt ab und zu als Berichterstatter bei hiesigen Journalen Verwendung gefunden hatte, agnosziert. Nahrungssorgen und die Furcht vor der Zukunft mögen den verheirateten Mann in den Tod getrieben haben. $\varkappa^{53}$

Der diese Notiz empfangende Chefredakteur - er heißt Dr. Wortmann - quittiert das mit »Es ist schrecklich - jetzt, wo der Mensch schreiben kann, jetzt erschießt er sich « ${ }^{54}$ und zahlt als erster in die Spendensammlung für die Witwe ein.

In Davids Novelle wird der suizidale Knalleffekt über kleine Andeutungen sorgfältig vorbereitet. Bernhofer versagt wie Werther an seiner Kunst, von der er empfindsam abschweift. ${ }^{55}$ Die oben erwähnte Subjektspaltung fällt auch hier wieder auf, daneben die Trennung zwischen Passagen in direkter Rede und Reflexionspassagen, an denen der allwissende Erzähler eingreifen muss; nur ist es hier ein von einer modernen Medienmaschine getriebener Suizident, der sich erst in seinen letzten Worten von der Schlacke des genialischen und empfindsamen Schreibmodells trennen kann, das selbst am Anfang der industrialisierten Medienmaschinerie steht.

\section{Literaturverzeichnis}

ALEXIS, Willibald (1843): Ein englischer Werther, in: Penelope. Taschenbuch auf das Jahr 1843, Leipzig 1843, S. 1-69.

ANDREE, Martin (2006): Wenn Texte töten: Über Werther, Medienwirkung und Mediengewalt, München.

BACHTIN, Michail M. (2017): Sprechgattungen, hg. v. Rainer Grübel, Renate Lachmann, Sylvia Sasse, übers. v. Alfred Sproede, Rainer Grübel, Berlin.

BEZ, Martin (2012): »... man hat uns photographiert«: Anmerkungen zu Heinrich Manns Novelle »Pippo Spano«, in: Heinrich-Mann-Jahrbuch, 30, 129-146.

BLANCKENBURG, Friedrich von (1774): Versuch über den Roman, Leipzig; Liegnitz.

BRANDSCH, Juliane, HENTSCHEL, Uwe, REK, Klaus, SCHUHMANN, Klaus, HARTINGER, Walfried (1995): Das literarische Leipzig. Kulturhistorisches Mosaik einer Buchstadt, hg. v. Andreas Herzog, Leipzig.

CROFT, Herbert (1780): Love and Madness. A story too True in a Series of Letters Between Parties, whose Names would perhaps be mentioned, were they less known, or less lamented, London.

CZEITSCHNER, Ulrike, KRAUTGARTNER, Barbara (2017): Discursive Constructions of Culture: Semantic Modelling for Historical Travel Guides, 5 (Apr, 2017) No 4, S. 323-331.

53 ebd, S. 189.

54 Ebd., S. 189.

55 Weitere Analogien sind allenfalls fußnotentauglich: Bernhofers Frau Helene trägt etwa Züge von Goethes Lotte. 
DAVID, Jakob Julius: Ein Poet? [Erstdruck, in vier Fortsetzungen erschienen] In: Arbeiter-Zeitung 9. 12. 1892, 10; 23. 12. 1892, 10; 30. 12. 1892, 6; 4. 6. 1. 1893, 9. URLs der Seitenbilder auf ANNO siehe oben Fußnote 52.

DAVID, Jakob Julius (1908): Ein Poet?, in: Gesammelte Werke, hg. v. Ernst Heilborn, Erich Schmidt, Bd. 3, München; Leipzig, 152-191.

FLASCHKA, Horst (1987): Goethes »Werther«, Werkkontextuelle Deskription und Analyse, München.

FOKKENS, Antske, BRAAKE, Serge ter, OCKELOEN, Niels, VOSSEN, Piek, LEGÊNE, Susan, SCHREIBER, Guus, BOER, Victor de (2017): BiographyNet: Extracting Relations Between People and Events: Aspekte, Bausteine, Normen und Standards für eine europäische Biographik, in: Europa baut auf Biographien, hg. v. Ágoston Zénó Bernád, Christine Gruber, Maximilian Kaiser, 1. Auflage, Wien, S. 193-223.

GENETTE, Gérard (2001): Paratexte. Das Buch vom Beiwerk des Buches, Frankfurt am Main.

GENETTE, Gérard (2010): Die Erzählung, übers. v. Andreas Knop, 3., durchgesehene und korrigierte Aufl., München.

GOETHE, Johann Wolfgang (1774): Die Leiden des jungen Werthers, Leipzig.

GOETHE, Johann Wolfgang (1999): Goethes Werke [Sophienausgabe], hg. v. im Auftrag der Großherzogin Sophie von Sachsen, unveränd. Nachdr. d. Ausg. Weimar, Böhlau, 1890, Weimar.

HOUBEN, Heinrich Hubert (1932): Der polizeiwidrige Goethe, Berlin.

JÄGER, Georg (1984): Die Leiden des alten und neuen Werther. Kommentare, Abbildungen, Materialien zu Goethes Leiden des jungen Werthers und Plenzdorfs Neuen Leiden des jungen W., München; Wien Literatur - Kommentare 21.

JOUFFROY, Théodore (1842): Nouveaux mélanges philosophiques par Theodore Jouffroy, hg. v. Jean Philibert Damiron, Paris.

KARTHAUS, Ulrich (1975): Zweihundert Jahre »Werther«, in: Gießener Universitätsblätter 2, S. 61-82.

KOCH, Herbert (1969): Johann Friedrich Weygand, Buchhändler in Leipzig. In: Archiv für Geschichte des Buchwesens, 9, S. 434-448.

KURZ, Stephan (2018): Der Briefroman des 18. Jahrhunderts als Gattungsproblem, in: Jahrbuch: Das Achtzehnte Jahrhundert und Österreich.

KURZ, Stephan: Gebundene Korrespondenzen. Gattungs- und Mediengeschichte von Briefromanen des 18. Jahrhunderts, Wien Univ. Diss.

LUDEWIG, Anna-Dorothea (2017): »Braut des hohen Liedes«: ’Jüdinnenbilder «im Werk von Leopold von Sacher-Masoch, in: Zagreber germanistische Beiträge, 26, S. 233-252.

MACHO, Thomas (2017): Das Leben nehmen: Suizid in der Moderne, Erste Auflage, Berlin.

MANN, Heinrich (1987): Künstlernovellen: Pippo Spano, Schauspielerin, Die Branzilla, Stuttgart (Universal-Bibliothek 8381).

MICHLER, Werner (1996): An den Siegeswagen gefesselt. Wissenschaft und Nation bei Wilhelm Scherer, in: Literatur und Nation. Die Gründung des Deutschen Reiches 1871 in der deutschsprachigen Literatur, hg. v. Klaus Amann, Karl Wagner, Wien; Köln; Weimar Literatur in der Geschichte, Geschichte in der Literatur 36, S. 233-266.

MICHLER, Werner (2015): Kulturen der Gattung. Poetik im Kontext, 1750-1950, Tübingen.

NUBER, Achim (2002): Neue Aspekte zu Arthur Schnitzlers Monolognovellen `Leutnant Gustl und `Fräulein Elseく. In: Zeitenwende - die Germanistik auf dem Weg vom 20. ins 21. Jahrhundert, Akten des X. Internationalen Germanistenkongresses Wien 2000. Epochenbegriffe: Grenzen und Möglichkeiten; Aufklärung - Klassik - Romantik; Die Wiener Moderne, hg. v. Peter Wiesinger, Uwe Japp, Ryozo Maeda, Helmut Pfotenhauer, John A. McCarthy, Al- 
bert Berger, Friedricht Vollhardt, Marijan Bobinac, Wendelin Schmidt-Dengler, Bern u.a., S. 427-432.

PECK, Clemens (2010): »Paralysis progressiva«. Zur Figuration des Bildungsproletariats in Jakob Julius Davids Wien-Roman Am Wege sterben. In: IASL 2, S. 37-60.

PICHLER, Caroline (1808): Agathokles, Wien.

RENNER, Ursula (2007): Dokumentation eines Skandals: Arthur Schnitzlers »Lieutenant Gustl«, in: Hofmannsthal-Jahrbuch zur europäischen Moderne, 15, S. 33-216.

RIEDL, Peter Philipp (2017): Renaissancemenschen: Literarische Konstruktionen eines Figurentyps um 1900. In: Der Renaissancismus-Diskurs um 1900, Bielefeld, S. 71-97.

SCHNITZLER, Arthur (1900): Lieutnant Gustl. In: Neue Freie Presse, 13053, S. 34-41.

SCHNITZLER, Arthur (2011): Werke in historisch-kritischen Ausgaben, hg. v. Konstanze Fliedl, Bd. 1, Berlin.

SCHNITZLER, Arthur Tagebuch: 1879-1931, hg. v. Werner Welzig, Peter Michael Braunwarth, Wien.

SEUFFERT, Bernhard (1900): Philologische Betrachtungen im Anschluß an Goethes Werther, in: Euphorion 7, S. 1-47.

THUMS, Barbara (2006): Die Macht der Bilder: oder der Kampf ums Dasein in Heinrich Manns Künstlernovelle »Pippo Spano«. In: Heinrich-Mann-Jahrbuch, 24, S. 45-60.

TIECK, Ludwig (1795/96): William Lovell, Berlin; Leipzig.

WEHDE, Susanne: Typographische Kultur. Eine zeichentheoretische und kulturgeschichtliche Studie zur Typographie und ihrer Entwicklung, Tübingen 2000.

WITTMANN, Reinhard (1995, 2003, 2012): Weygand - der »Fabrikbuchhändler«, in: Das literarische Leipzig. Kulturhistorisches Mosaik einer Buchstadt, hg. v. Andreas Herzog, Leipzig, S. 126-127.

A companion to the works of Arthur Schnitzler, hg. v. Dagmar C. G. Lorenz, Rochester, NY u.a. »A. ist manchmal wie ein kleines Kind«. Clara Katharina Pollaczek und Arthur Schnitzler gehen ins Kino, hg. v. Stephan Kurz, Michael Rohrwasser, Daniel Schopper, Wien.

\section{Mag. Dr. Stephan Kurz / stephan.kurz@oeaw.ac.at}

Österreichische Akademie der Wissenschaften, Forschungsbereich Kulturelles Erbe: Biographik und Editionen, Hollandstraße 11-13, 1020 Wien, AT 